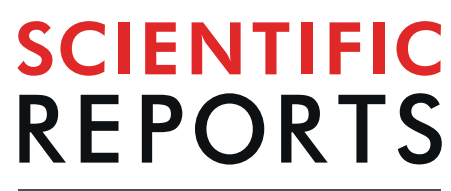

natureresearch

\title{
Oral treatment with T6-loaded yeast cell wall particles reduces the parasitemia in murine visceral leishmaniasis model
}

\author{
Débora B. Scariot ${ }^{1,6}$, Hélito Volpato ${ }^{1,6}$, Nilma S. Fernandes $\left(\mathbb{1}^{2}{ }^{2}\right.$, Danielle Lazarin-Bidóia $\mathbb{1}^{1}$, \\ Olga Borges ${ }^{3,4}$, Maria do Céu Sousa ${ }^{3,4}$, Fernanda A. Rosa ${ }^{5}$, Andrey P. Jacomini ${ }^{5}$, Sueli O. Silva ${ }^{1}$, \\ Tânia Ueda-Nakamura ${ }^{1}$, Adley F. Rubira ${ }^{5}$ \& Celso V. Nakamura ${ }^{1 *}$
}

Yeast cell wall particles isolated from Saccharomyces cerevisiae (scYCWPs) have a rich constitution of $\beta$-glucan derived from the cell wall. After removing intracellular contents, $\beta$-glucan molecules are readily recognized by dectin-1 receptors, present on the cytoplasmic membrane surface of the mononuclear phagocytic cells and internalized. Leishmania spp. are obligate intracellular parasites; macrophages are its primary host cells. An experimental murine model of visceral leishmaniasis caused by $L$. infantum was used to evaluate the antileishmanial activity of oral administration of these particles. A low-water soluble thiophene previously studied in vitro against $L$. infantum was entrapped into scYCWPs to direct it into the host cell, in order to circumvent the typical pharmacokinetic problems of water-insoluble compounds. We found that sCYCWPs + T6 reduced the parasitic burden in the liver and spleen. There was an increase in IFN- $\gamma$ levels related to nitric oxide production, explaining the reduction of the $L$. infantum burden in the tissue. Histological analysis did not show signals of tissue inflammation and biochemical analysis from plasma did not indicate signals of cytotoxicity after scYCWPs + T6 treatment. These findings suggested that scYCWPs + T6 administered through oral route reduced the parasitic burden without causing toxic effects, satisfying requirements for development of new strategies to treat leishmaniasis.

In the last decade, technological advances have enabled a revolution in the treatment of medical conditions, from gene therapy and personalized medicines to 3D-printed body parts. However, when it comes to neglected tropical diseases, the advances have moved forward much more slowly. Between 2008 and 2014, only 1.5\% of the novel products starting the phase-I trial were aimed at the prevention or treatment of neglected tropical diseases $^{1}$ - among them, leishmaniasis. Despite the collaborative efforts and financial support of the World Health Organization (WHO), research institutes, pharmaceutical companies, and entrepreneurs ${ }^{2,3}$, the WHO estimates that up to 1 million new cases and 65,000 deaths continue to occur annually for leishmaniasis worldwide (https:// www.who.int/news-room/fact-sheets/detail/leishmaniasis). The major challenges in the drug discovery and development for the treatment of leishmaniasis is not just related to the cost required for research but the biology of the parasite itself. Leishmania spp. are obligate intracellular parasites, which reside in effector cells of the mononuclear phagocyte system, therefore new antileishmanial molecules must be able to reach the parasites without promoting host cell damage ${ }^{2,4,5}$. The high toxicity and severe side-effects associated with current antileishmanial drugs often leads to treatment abandonment by patients and thus the development of drug-resistant Leishmania strains, highlighting a need for alternative therapies ${ }^{6}$.

Leishmania spp. can evade the host immune response by sequestering itself inside innate immune cells and inhibiting the actions of these cells that promote parasite death, such as the production of nitric oxide and

${ }^{1}$ Laboratório de Inovação Tecnológica no Desenvolvimento de Fármacos e Cosméticos, State University of Maringá, Maringa, 87020-900, Brazil. ' Cellular Biology Graduate Program, State University of Maringá, Maringa, 87020-900, Brazil. ${ }^{3}$ Faculty of Pharmacy, University of Coimbra, Coimbra, 3000-548, Portugal. ${ }^{4} \mathrm{CNC}$ - Center for Neurosciences and Cell Biology, University of Coimbra, Coimbra, 3000-548, Portugal. ${ }^{5}$ Chemistry Department, State University of Maringá, Maringa, 87020-900, Brazil. ${ }^{6}$ These authors contributed equally: Débora B. Scariot and Hélito Volpato.

*email: cvnakamura@gmail.com 
pro-inflammatory cytokines. In addition, Leishmania spp. can induce the secretion of immunosuppressive molecules through to the late phase of the disease ${ }^{7}$. Therefore, restoring the regular host immune response through immunomodulatory molecules could be a potential approach to control the parasitic burden and the relapse of leishmaniasis ${ }^{2,6}$.

$\beta$-glucan, a polysaccharide present in the cell wall of bacteria, protists, and fungi is a well-known natural immunostimulant, used as an adjuvant in vaccines and as immunotherapy against tumors ${ }^{8-11}$. In Japan, $\beta$-glucan has been used to treat malignancies for decades ${ }^{8,12,13}$ and some studies suggest that $\beta$-glucan is also active against intracellular pathogens improving the host immune response against Leishmania spp., Plasmodium spp., Listeria monocytogenes, and Toxoplasma gondii ${ }^{14,15}$. $\beta$-glucan from the yeast Saccharomyces cerevisiae is also considered completely non-toxic and safe, and is used as an oral prebiotic and has been used in the human diet since early civilizations as a constituent of the baker's powder and brewer's yeast ${ }^{14,15}$.

The cell walls of $S$. cerevisiae consist of mannoproteins, $\beta$-glucan, and chitin, which are linked covalently, providing a stable structure that preserves the cell's osmotic integrity and morphology ${ }^{16}$. A hot alkaline hydrolysis process removes the alkali-soluble content from the yeast cell wall as well as the intracellular material. This leaves the alkaline-insoluble material resulting in "ghost" or hollow $\beta$-glucan spheres with an inner cavity where it is possible to entrap active molecules ${ }^{17-19}$. Particles obtained from Saccharomyces cerevisiae yeasts can be called scYCWPs (yeast cell wall particles isolated from S. cerevisiae) and can be used to carry the encapsulated molecules directly to target cells for the purposes of treatment ${ }^{19}$. Saccharomyces bayanus, Candida utilis, Kluyveromyces fragilis, Cryptococcus curvatus, Endomyces vernalis among others microorganisms have also been used as sources of YCWPs $^{20}$. Currently, there is a clinical trial (phase I/II) using YCWPs in a vaccine to treat metastatic melanoma (https://clinicaltrials.gov/ct2/show/NCT02678741).

As a drug carrier, $s c$ YCWPs can deliver low-water soluble molecules into cells of the mononuclear phagocyte system, such as monocytes, dendritic cells, and macrophages. scYCWPs are biodegradable and biocompatible and their rich constitution of $\beta$-glucan allows an efficient and rapid recognition by macrophages and dendritic cells via receptor dectin-1-mediated endocytosis ${ }^{21-23}$. As a biodegradable material, scYCWPs could be digested intracellularly via host-oxidative pathways, releasing the entrapped molecule and $\beta$-glucan fragments ${ }^{24}$. Volpato et al. ${ }^{25}$ recently showed the promising antileishmanial activity of empty scYCWPs and $s c Y C W P s$ containing the synthetic compound thiophene (T6) (4-[(2E)- $N^{\prime}-\left(2,2^{\prime}\right.$-bithienyl-5-methylene)hydrazinecarbonyl]-6,7-dihydro-1-phenyl-1Hpyrazolo[3,4-d]pyridazin-7-one) on an in vitro model of Leishmania infantum-infected macrophages. Furthermore, studies suggest that after oral administration, $s c$ YCWPs accumulate in intestinal Peyer's patches, inside macrophages or M-cells, which move towards the Leishmania-infected target organs, such as the bone marrow, spleen, and lymph nodes, through the bloodstream ${ }^{17,26}$. Electronic micrographs in previous in vitro studies demonstrated that the scYCWPs phagocytosed by Leishmania-infected macrophages can be located within the same vacuoles after the fusion between the phagosome and the parasitophorous vacuoles mediated by lysosomes action ${ }^{27-30}$.

Based on the findings by Volpato et al. ${ }^{25}$, the current study was proposed to investigate the efficacy of the T6-loaded immunomodulatory carrier ( $s c$ YCWPs + T6) in reducing the parasitic load in the visceral leishmaniasis murine model and sought to better understand how the treatment affected the in vivo host response. We demonstrate that oral administration of T6-loaded $s c$ YCWPs reduces the splenic and hepatic parasitic burden of L. infantum in mice by increasing the level of IFN- $\gamma$ without promoting toxic effects. Our findings also indicate an immunomodulatory effect of the $s c$ YCWPs alone as a key for the antileishmanial activity of T6-loaded $s c$ YCWPs.

\section{Results}

Treatments did not cause changes in body mass, but altered spleen mass of the mice. Neither $\mathrm{BALB} / \mathrm{c}$ mice that were noninfected nor those that were infected with $L$. infantum (Fig. 1a,b) showed significant variations in body mass during 21 days of treatment compared to control groups. Between the first and last day of treatment, the body mass variation was compatible with advancing age. However, on the last day of treatment, there was a significant difference in the body mass between infected animals treated with scYCWPs + T6 and T6 alone (Fig. 1b). In terms of organ masses, the noninfected animals did not present significant differences (Fig. 1a). Compared with the untreated infected mice, the liver of animals treated with the empty scYCWPs was the only organ that had lower mass among infected mice (Fig. 1b) whilst infected animals treated with scYCWPs + T6 had higher spleen mass. The masses of the kidneys, lungs and hearts did not change within the infected animals, treated or not (Fig. 1b).

T6-loaded scYCWPs reduced the $L$. infantum burden in the spleen and liver. Quantitative data regarding the L. infantum parasitic burden in organs were obtained using the qPCR method. As expected, the parasitic burden was significantly lower in the spleen and liver after treatment with the antileishmanial drug, miltefosine. Compared to the untreated control, scYCWPs + T6 caused an 85\% reduction in the number of parasites in the spleen $(p=0.0008)$, and the empty scYCWPs and T6 alone did not promote a significant decrease in the number of splenic parasites (Fig. 2a). Furthermore, the T6-loaded scYCWPs showed significantly greater antileishmanial activity than the T6 alone $(p=0.0479)$, although there was no statistical difference between the empty scYCWPs and $s c$ YCWPs + T6. In the liver, $75 \%$ of the animals treated with $s c$ YCWPs + T6 had significant reduction in the parasite burden in comparison to the untreated mice (Fig. 2b), representing a 15-fold reduction in parasite number $(p=0.0171)$. As in the spleen, empty $s c$ YCWPs and T6 alone were not able to cause a significant reduction in the hepatic parasitism in comparison to untreated control and $s c$ YCWPs + T6 treated group.

Splenic IFN- $\gamma$ and TNF- $\alpha$ levels increased after treatment of infected mice with scYCWPs and T6 loaded-scYCWPs. To assess the immunomodulatory action of treatment, the cytokine milieu in the spleens was evaluated. In noninfected animals, the treatment with empty scYCWPs caused a decrease in TNF- $\alpha$ 
a
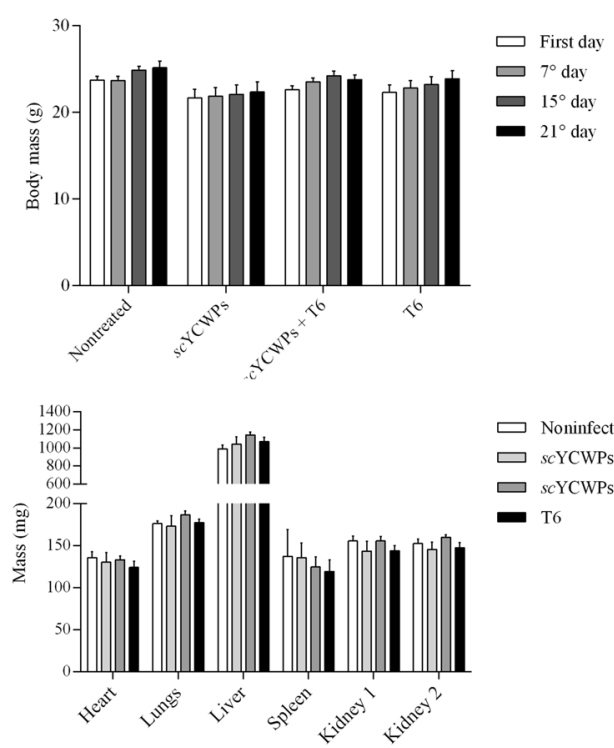

$\square$ scYCWPs

T6 b

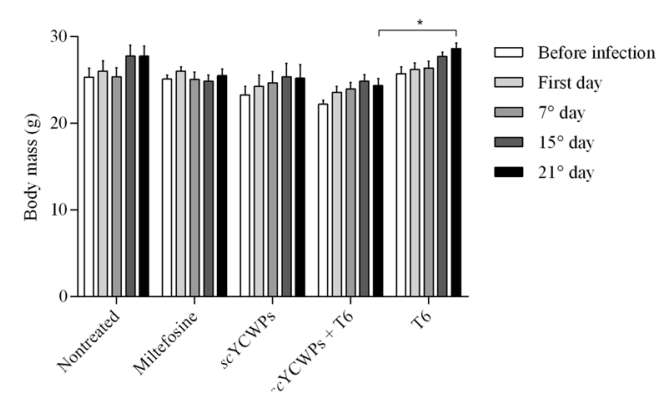

$\square$ Noninfected contr

$\square$ scYCWPs + T6

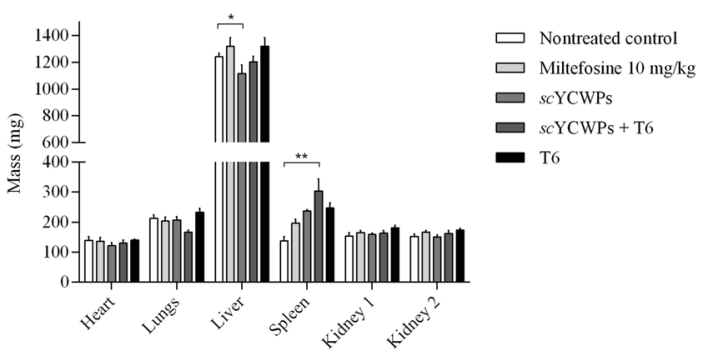

Figure 1. Body mass over the course of treatment and organs mass at the last day of treatment of noninfected (a) and L. infantum-infected (b) mice. Animals were treated with empty scYCWPs (scYCWPs), T6-loaded scYCWPs (scYCWPs + T6), T6 alone (T6), and miltefosine was used as standard drug against visceral leishmaniasis. Results were expressed as mean \pm standard error $(\mathrm{n}=4)$. Significant differences at $* p<0.05$ and $* * p<0.01$.
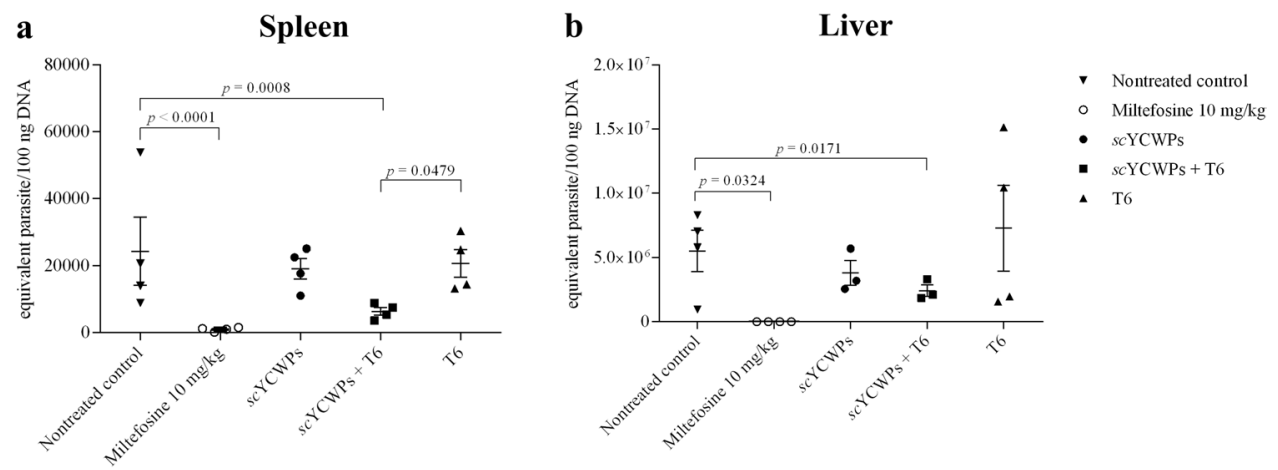

Figure 2. L. infantum burden in the spleen (a) and liver (b) determined by qPCR after treatment with empty scYCWPs (scYCWPs), T6-loaded scYCWPs (scYCWPs + T6), and T6 alone (T6). Miltefosine was used as the antileishmanial activity control. Each point represents one animal $(n=4)$. At least $75 \%$ of the mice were considered in the statistical analysis. Significant differences and $p$ values are represented in the graph space.

compared to those that were untreated. However, the T6-loaded scYCWPs were responsible for causing a significant rise in the TNF- $\alpha$ level compared with the untreated control and animals treated with empty scYCWPs (Fig. 3a).

In infected animals, a different cytokine profile was observed, particularly in the case of IFN- $\gamma$ secretion, with empty $s c$ YCWPs, $s c$ YCWPs + T6, and T6 alone promoting a similar significant increment in the IFN- $\gamma$ levels. With regards to the TNF- $\alpha$ level in the infected mice, treatment with the empty scYCWPs showed a significant TNF- $\alpha$ increase in comparison to the untreated control. Whilst treatment with scYCWPs + T6 was not significantly higher than the untreated control, the level lied between the TNF- $\alpha$ overproduction from empty scYCWPs and the underproduction from T6 treatment (Fig. 3b). With the exception of IFN- $\gamma$ levels, treatment with miltefosine did not significantly alter cytokine levels in comparison with the untreated control. No significant variations were observed for IL-2, IL-4, IL-6, IL-10, IL-17A in all groups of infected and uninfected mice.

Treatment with T6-loaded scYCWPs does not show biochemical signs of toxicity. Biochemical analyses of the plasma of noninfected animals showed the preservation of renal, hepatic and cardiac functions after treatments (Fig. 4a). The creatinine level, an indicator for renal dysfunction, was significantly higher in the untreated infected group compared to the noninfected control and the infected animals treated with 

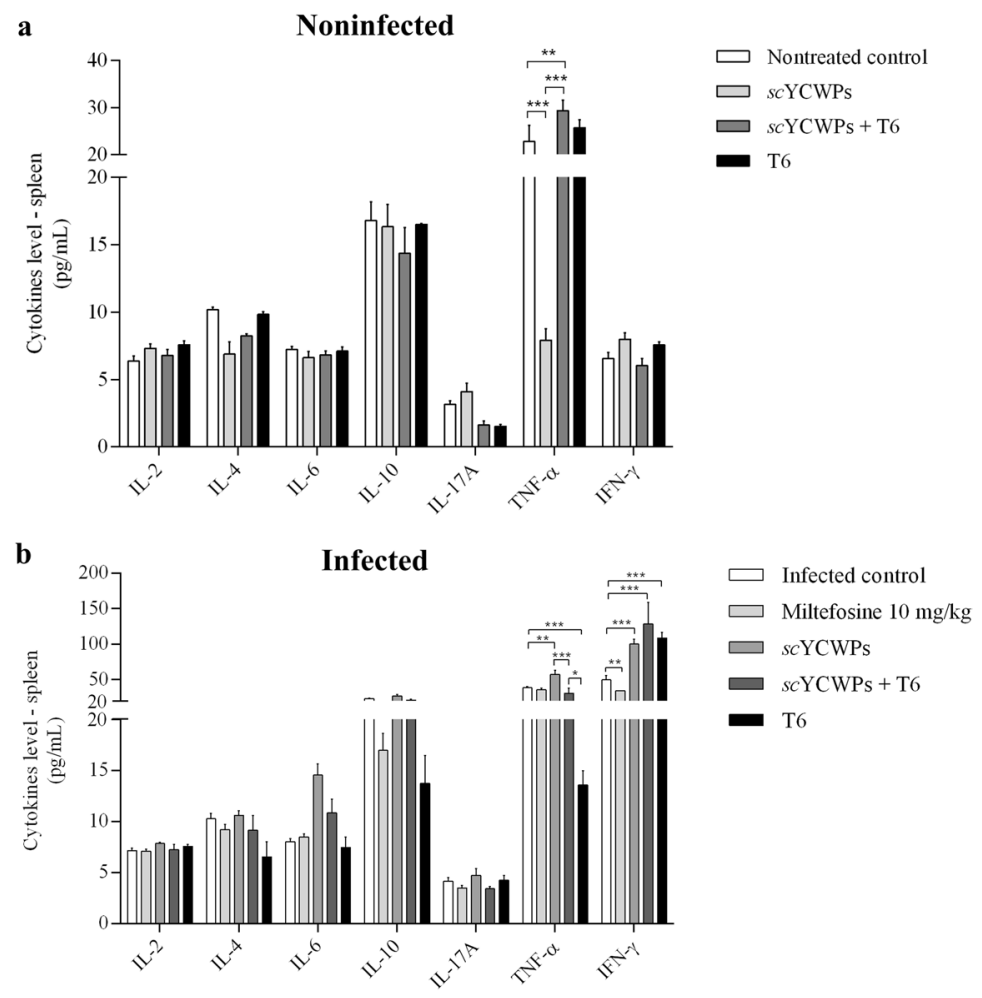

Figure 3. Cytokine measurements from the spleens of noninfected (a) and L-infantum infected (b) mice after treatment with empty scYCWPs (scYCWPs), T6-loaded scYCWPs (scYCWPs + T6) and T6 alone (T6). Miltefosine was used as the standard drug against visceral leishmaniasis. Data were expressed as cytokine production in $\mathrm{pg} / \mathrm{ml}$ and shown as mean \pm standard error $(\mathrm{n}=4)$. Using the BD Cytometric Bead Array $(\mathrm{CBA})$ Mouse Th1/Th2/Th17 Cytokine Kit manufacturer's instruction, 2,500 events were analyzed for each sample. Significant differences at $* p<0.05, * * p<0.01, * * * p<0.001$.

scYCWPs + T6, T6 alone, and miltefosine. Biomarkers of liver damage, AST and ALT, revealed hepatic abnormalities in infected animals only after treatment with the empty scYCWPs in comparison to the other groups, including the untreated mice (Fig. 4b). Treatments did not cause a significant change in cardiac function, according to biochemical analysis of CKMB.

\section{Discussion}

The treatment of leishmaniasis has been minimally changed since the 1920s when pentavalent antimonials were first made available ${ }^{31}$. Severe side effects, low efficacy, development of antimony-resistant strains, and the requirement of drug administration to occur exclusively in the hospital environment are clear motivations for the discontinuation of this treatment ${ }^{31}$. However, while there is a clear need for therapeutic innovation, some obstacles must be overcome early in the drug discovery process. At the laboratory research level, the low water solubility of bioactive molecules impairs the in vivo studies by compromising pharmacokinetic parameters, including absorption, permeability and consequently bioavailability. Entrapment of these compounds in liposomes, nanoparticles, microparticles, hydrogels or microemulsions are techniques to improve the solubility, and consequently, the effectiveness of drugs $\mathrm{s}^{32,33}$. However, the high cost of this technology could hinder the access to certain populations, particularly inhabitants of developing countries ${ }^{34-37}$. Liposomal amphotericin B (AmBisome) is a relevant example of this; despite a successful private-public partnership led by WHO, which has reduced the prices for AmBisome for the public sector of the eligible low-income countries, the cost of liposomal amphotericin B and hospitalization required for the parenteral drug administration are still high for the most Leishmania endemic countries $^{38-40}$. Therefore, the search for new molecules or strategies to combat this disease should focus on four major requirements: enhancing leishmanicidal activity, minimizing toxicity, supporting oral administration, and being price compatible with the economic reality of the tropical endemic countries. Considering all these points, we report here the reduction of $L$. infantum burden in experimental murine visceral leishmaniasis after oral treatment with an innovative low water-soluble thiophene T6 entrapped in immunomodulatory glucan particles (scYCWPs) obtained from barker's powder, an affordable raw material.

Spleen and liver are the most affected organs in visceral leishmaniasis, which is most severe form of the leishmaniases. Independent of the causative visceralizing Leishmania species, the amastigote burden in the mammalian host persists in the spleen during the entire infection period ${ }^{41,42}$. Thus, splenic parasite reduction is fundamental to successful therapy. In the experimental model used in this investigation, the T6-loaded scYCWPs treatment reduced the L. infantum splenic burden. The ineffectiveness in controlling L. infantum burden after treatment with the empty scYCWPs and T6 alone suggests that the presence of both is fundamental for the 
a
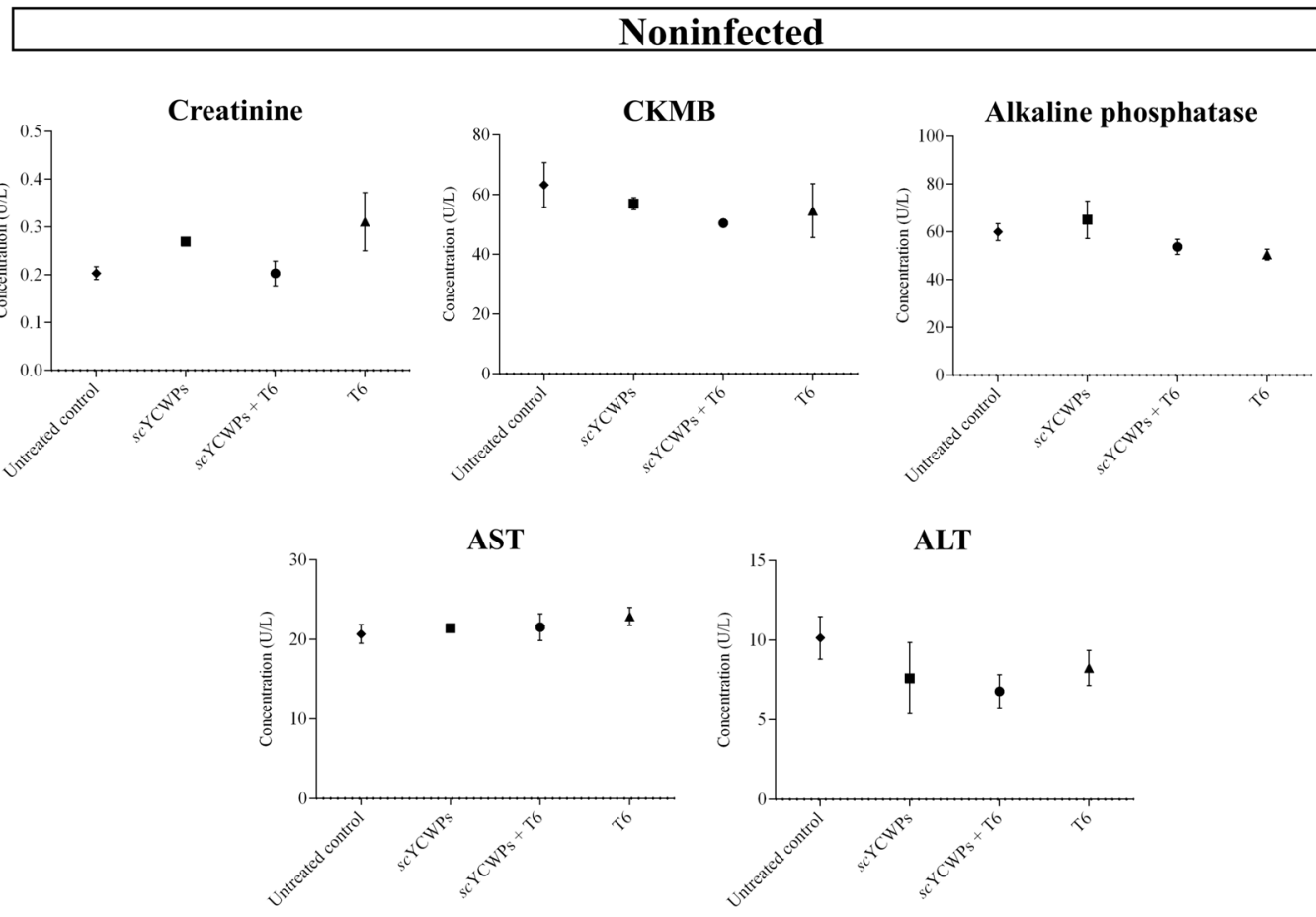

b
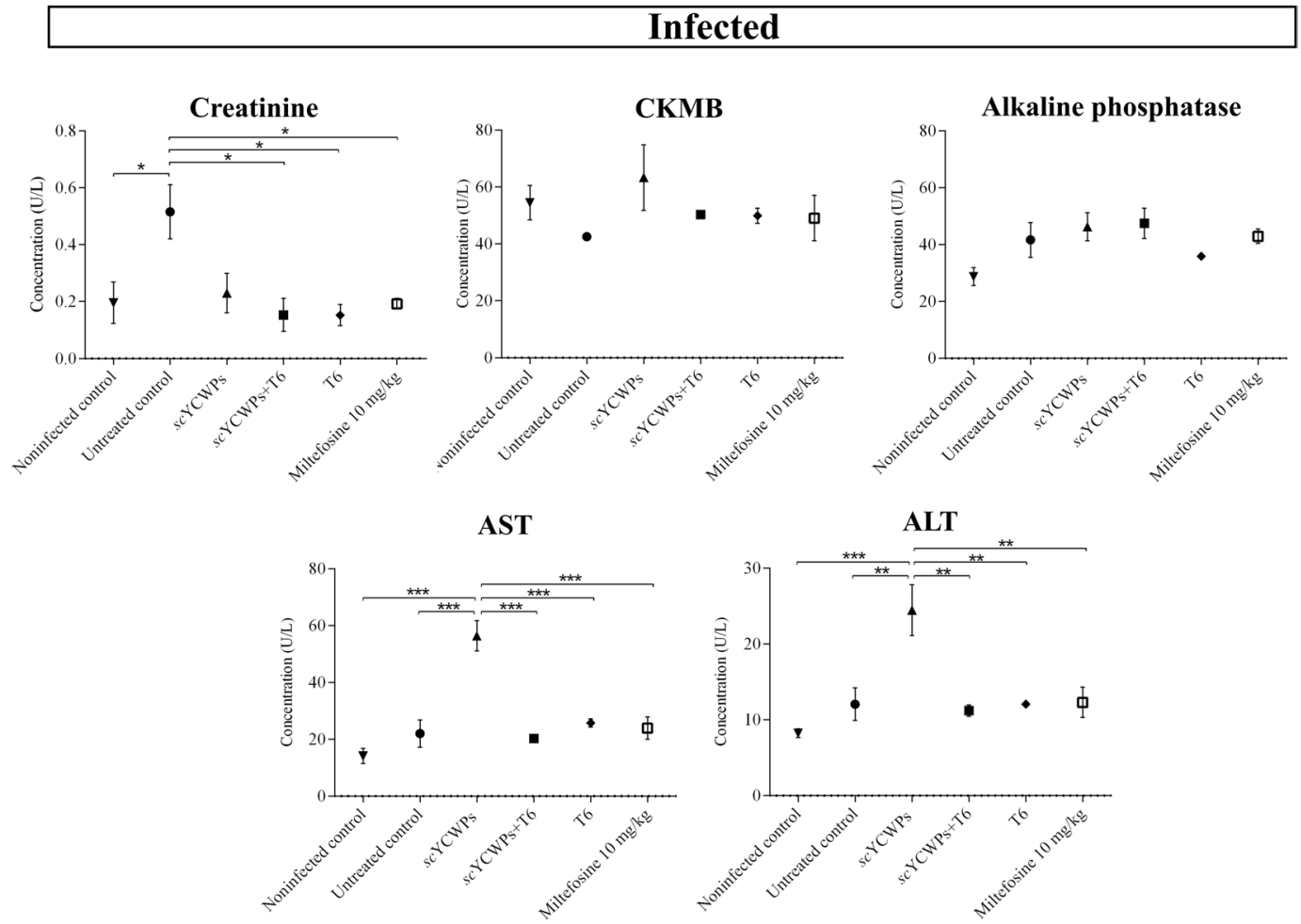

Figure 4. Biochemical analysis of plasma from (a) noninfected and (b) L. infantum-infected animals. In both assays, animals were treated with empty scYCWPs (scYCWPs), T6-loaded scYCWPs (scYCWPs + T6), and T6 alone (T6). Miltefosine was used as the standard antileishmanial drug to treat infected mice. Renal, cardiac and hepatic functions were measured through creatinine, CKMB, and alkaline phosphatase, AST and ALT levels, respectively, by UV kinetic determination. All tests were performed in duplicate with three animals per group and the results were shown as mean \pm standard error. Significant differences at $* p<0.05, * * p<0.01$, $* * * p<0.001$.

antileishmanial activity. scYCWPs may have worked as a protector structure for the antileishmanial molecule T6, as suggested by Ren et al. ${ }^{43}$, which demonstrated that $s c$ YCWPs could avoid gastrointestinal degradation of entrapped molecules. 
The hepatic infection, on the other hand, is usually self-resolving after 6-8 weeks of infection ${ }^{44}$. However, there is not complete elimination of parasites as their presence is essential for long-term immunity ${ }^{45}$. In our study, the standard drug miltefosine promoted the parasite clearance from liver almost completely and $s c$ YCWPs + T6 treatment was able to cause a significant parasite burden reduction but a high number of parasites still remained. Higher doses of $s c$ YCWPs + T6 or a longer treatment period could improve the hepatic L. infantum clearance. In the liver, amastigote-controlled growth is determined by the phagolysosome proton pump of macrophages, encoded by the SLc1lal gene, creating $\mathrm{Fe}^{2+}, \mathrm{Zn}^{2+}, \mathrm{Mn}^{2+}$ deprived conditions inside phagolysosomes. These cations are essential for enzymatic processes of the parasite related to growth, virulence, and protection against host oxidative stress. A mutation in the SLc11al gene, resulting in pump dysfunction, is responsible for the susceptibility of the $\mathrm{BALB} / \mathrm{c}$ mice to visceral leishmaniasis, raising the hepatic parasitic burden up to 100 -fold ${ }^{46,47}$. This genetic condition may explain the high parasite number in the liver observed in this study.

scYCWPs have great potential as an oral drug delivery system as they are biodegradable and biocompatible and our results show delivery to the target organs in this disease model. The rich constitution of $\beta$-glucan in the scYCWPs allows an efficient and rapid recognition by macrophages and dendritic cells via receptor dectin-1-mediated endocytosis. As a biodegradable material, scYCWPs can be digested intracellularly via host-oxidative pathways, releasing the entrapped molecule and $\beta$-glucan fragments. Unlike bone marrow, spleen, and lymph nodes, there is no consensus about whether the $s c$ YCWPs are brought to the liver ${ }^{43,48,49}$, but our findings may indicate that this delivery to the liver occurs since the L. infantum hepatic burden presented significant reduction after oral administration of T6-loaded $s c$ YCWPs.

Each Leishmania species and strain, which cause the spectrum of the disease, generates a dynamic profile of inflammation in vivo with different patterns of inflammatory mediators secretion ${ }^{50}$. In general, self-resolving infections are marked by a balance of Th- 1 and Th- 2 immune responses. An exacerbated pro-inflammatory Th-1 response, with high IFN- $\gamma$ production, is typical of lesions with low healing capacity, as well as of deforming mucocutaneous wounds. Chronic and anergic leishmaniasis are characterized by an intense anti-inflammatory Th-2 response ${ }^{51}$. In this study there were substantial changes in the splenic IFN- $\gamma$ concentrations after treatments with the empty scYCWPs, scYCWPs + T6, and T6 alone. Typically, IFN- $\gamma$ secretion is induced by TNF- $\alpha$, which in turn induces the Th-1 response. IFN- $\gamma$ is responsible for up-regulating the inducible nitric oxide synthase (iNOS) from L-arginine, releasing large amounts of nitric oxide (NO), responsible for killing intracellular para$\operatorname{sites}^{52}$. However, recent studies showed that high concentrations of IFN- $\gamma$ were insufficient to control Leishmania infection in TNF-deficient mice, suggesting the need for basal TNF- $\alpha$ production and a NO effect ${ }^{53}$. It is worth noting that high NO production is considered fundamental for the resolution of leishmaniasis; however, NO production is not completely dependent on TNF- $\alpha$ secretion $^{52,54}$. This could be observed in this investigation because the increase of IFN- $\gamma$ levels after treatment with $s c$ YCWPs + T6 was independent of an increase in TNF- $\alpha$. However, TNF- $\alpha$ levels after treatment with T6 were lower than the untreated control which may be related to the failure of T6 alone to control the infection, despite the increase IFN- $\gamma$.

Some previous studies showed that the standard leishmanicidal drug miltefosine may act as an immunomodulatory molecule, stimulating IL-12 secretion and, consequently, increasing IFN- $\gamma$ and NO. However, these findings depend on several parameters, such as the Leishmania species, animal model or type of cell, administration route, and dose considered in the studies ${ }^{55}$. In our model, the data suggest that miltefosine acts via an inherent effect on the protozoan, without altering cytokines level. This can be corroborated by previous findings, which revealed the effectiveness of miltefosine in immunodeficient mice and HIV-coinfected patients, indicating that its activity is independent of a T and B-cell mediated immune response ${ }^{56-59}$. Alternatively, miltefosine could alter the intracellular lipid metabolism of parasite, acting directly on the parasite ${ }^{60}$.

The absence of inflammation signals in splenic and hepatic tissue of animals treated with T6 alone, suggests that T6 may have reduced the inflammation and, consequently, promoted high parasite growth (Supplementary Figs. S1 and S2). These findings can reinforce the splenic tissue patterns verified by Cavancanti et al. ${ }^{61}$ in $L$. infantum-naturally infected dogs. Although hepatic granulomas precede the self-resolution of the parasitism in immunocompetent hosts, excessive inflammation may cause tissue damages and toxic symptoms ${ }^{62}$. Empty scYCWPs did not promote a significant parasite decrease in the liver, but extensive hepatic granulomas could be observed in the histological analysis (Supplementary Fig. S2) as well as hepatic biomarkers (AST/ALT) revealed a discrete liver commitment.

In summary, T6-loaded $s c$ YCWPs unite the immunomodulatory effect of the $s c$ YCWPs and the active compound T6, creating an equilibrium between effectiveness and safety. The $s c Y C W P s+\mathrm{T} 6$ treatment resulted in reduced parasite burdens in the absence of toxic signals or exacerbated inflammation in the spleen and liver of $L$. infantum-infected animals. Our findings emphasize the application of $s c$ YCWPs as a drug microcarrier for oral administration. The low-cost raw material used in this study contrasts with the high cost of polymers commonly used for the development of drug delivery systems. The scYCWP as a drug delivery system exhibits great potential for future drug discovery investigations against others intracellular pathogens.

\section{Materials and Methods}

Chemicals. Saccharomyces cerevisiae (baker's yeast) was obtained from Mauripan Instant Dry Yeast. RPMI 1640 medium and fetal bovine serum were purchased from Gibco - Life Technologies Corporation. Miltefosine, poly-L-lysine, and acetone (analytical grade) were purchased from Sigma-Aldrich. PureLink Genomic DNA Mini Kit and Leishmania kDNA primers were obtained from Invitrogen and Integrated DNA Technologies (Coralville, IA, USA), respectively. Platinum SYBRGreen from Thermo Fisher Scientific. Permount mounting medium was purchased from Fisher Chemical and yellow eosin from InLab (São Paulo, Brazil). Histological paraffin, ethanol (analytical grade), and xylol (analytical grade) were obtained from Synth. Harris's hematoxylin and biochemical kits for quantification of creatinine, ALT, AST, CKMB, and alkaline phosphatase were purchased 
from Laborclin - Bio Liquid. BD Cytometric Bead Array (CBA) Mouse Th1/Th2/Th17 Cytokine Kit was purchased from BD Bioscience (Becton, Dickinson and Company).

Preparation and characterization of the empty scYCWPs and scYCWPs + T6. Empty $s c Y C W P s$ and $s c$ YCWPs + T6 were obtained according to Volpato et al. ${ }^{25}$. Briefly, $20 \mathrm{~g}$ of $S$. cerevisiae powder underwent to a hot acid-base extraction using $\mathrm{NaOH} 1 \mathrm{M}$ and $\mathrm{HCl} 1 \mathrm{M}$, at $85^{\circ} \mathrm{C}$. At the end of the process, the insoluble material was collected by centrifugation $(2000 \times \mathrm{g}$ for $10 \mathrm{~min})$ and washed in water, isopropanol, and acetone. The residual acetone was removed by evaporation at room temperature. This process allowed to obtain the $s c$ YCWPs, rich glucan-content, by removing all intracellular structures, as well as the proteins, lipids, and mannan from the cell wall. To entrap T6 in the $s c$ YCWPs, $100 \mu \mathrm{L}$ of T6 solution at $500 \mu \mathrm{g} / \mathrm{mL}$ in acetone were added in the $10 \mathrm{mg}$ of empty scYCWPs, and incubated at $-20^{\circ} \mathrm{C}$, for $2 \mathrm{~h}$. Next, the acetone was removed by evaporation at room temperature. This sequence was repeated 5 times to promote the T6 accumulation inside scYCWPs. The entrapment efficiency was determined by quantifying of the not entrapped T6 on spectrophotometer (Shimadzu UV-1700 PharmaSpec), at $382 \mathrm{~nm}$. scYCWPs and scYCWPs + T6 were characterized by Volpato et al., through of transmission electron microscopy, and zeta potential, Fourier transform infrared (FT-IR), and X-ray diffraction analysis ${ }^{25}$.

Infective parasite maintenance. Leishmania infantum MCAN/BR/97/P142 (syn. L. chagasi) promastigotes in a stationary phase of growth (7-8 days) were inoculated intraperitoneally in a female BALB/c mouse. After 30 days, the spleen was removed and maintained in RPMI 1640 medium over 15 days, when infective metacyclic promastigote forms were detected in the culture supernatant. These infective parasites were maintained in RPMI 1640 medium supplemented with $10 \%$ of fetal bovine serum (FBS). After the first passage, the parasites were in the stationary phase by the eighth day.

Visceral leishmaniasis murine model. Female BALB/c mice (7-8 weeks old) were infected with L. infantum promastigotes at the stationary phase of growth, obtained as described above. A suspension containing $1 \times 10^{8}$ promastigotes $/ \mathrm{mL}$ in $0.01 \mathrm{M}$ PBS was inoculated intraperitoneally. Each group of mice was kept in a separate cage (four animals per cage), at room temperature $\left(25 \pm 1^{\circ} \mathrm{C}\right)$, and at a 12 -hour light-dark cycle. Animals received water and food ad libitum. At the end of 30 days post-infection, treatment was started and lasted 21 days. The infected animals were divided into 5 groups: non-treated animals; treated with $10 \mathrm{mg}$ of miltefosine $/ \mathrm{kg} / \mathrm{day}$; treated with $3 \mathrm{mg}$ of empty $s c \mathrm{YCWPs} / \mathrm{mouse}$; treated with $3 \mathrm{mg}$ of $s c \mathrm{YCWPs} / \mathrm{mouse}$ containing $45.7 \mu \mathrm{g}$ of T6 (or $15.23 \mu \mathrm{g}$ of $\mathbf{T 6} / \mathrm{mg} s c \mathrm{YCWPs}$ ); and treated with $45.7 \mu \mathrm{g}$ of free T6/mouse (equivalent to that containing in $3 \mathrm{mg}$ of $s c$ YCWPs + T6). The mass of T6 contained in the scYCWPs was determined in previous study by Volpato et $a l$., according to the encapsulation efficiency data ${ }^{25}$. All tested treatments used water as the vehicle. Noninfected animals were used as negative controls. The mice were weighed once a week. On the last day of the treatment, the animals were euthanized using a lethal dose of isoflurane and oxygen. Blood was collected by cardiac puncture and, after centrifugation, plasma was isolated and stored. Heart, lungs, spleen, liver and kidneys were collected, weighed and stored for further analysis. In an additional assay, female BALB/c mice (7-8 weeks old) were maintained healthy and received the same treatment as the infected mice (except for miltefosine treatment), in order to evaluate the basal response caused by the treatments.

Ethics statement. All animal experiments were approved by the Ethical Committee on the Use of Animals (CEUA) from State University of Maringá (Protocol number 1323011116), following the requirements provided by the Regulatory Resolutions numbers 33/2016 and 39/2018 of the Ministry of Science, Technology, Innovations and Communications of Brazil.

Quantitative real-time PCR. Spleen and liver were used to quantify the parasitic load. DNA was extracted from the tissues using the PureLink Genomic DNA Mini Kit, according to the manufacturer's instructions. Briefly, about 10 and $25 \mathrm{mg}$ of spleen and liver, respectively, were weighed and mixed with digestion buffer and proteinase $\mathrm{K}$. After $4 \mathrm{~h}$ in a hot water bath, the samples were centrifuged at maximum rotation, adding RNase A and lysis buffer. DNA was precipitated in ethanol, purified in spin columns, washed and eluted in elution buffer. Real-time PCR was performed using the LightCycler 480 system (Roche Diagnostics Brussels, Belgium) according to the manufacturer's instructions. Reactions were performed in a $20 \mu \mathrm{L}$ final volume with $300 \mathrm{nM}$ of Leishmania kDNA specific forward primer 5'-CTTTTCTGGTCCTCCGGGTAGG-3' and reverse primer 5'-CCACCCGGCCCTATTTTACACCAA- ${ }^{\prime}$, previously described ${ }^{63}$ and Platinum SYBRGreen qPCR. Each PCR reaction contained $100 \mathrm{ng}$ genomic tissue DNA. The amplification protocol consisted of a denaturation phase at $95^{\circ} \mathrm{C}$ for $10 \mathrm{~s}$, then 35 cycles of amplification $95^{\circ} \mathrm{C}$ for $15 \mathrm{~s}, 60^{\circ} \mathrm{C}$ for $60 \mathrm{~s}$. At the end of each run, melting curve analysis was performed from $65^{\circ} \mathrm{C}$ to $95^{\circ} \mathrm{C}$ to monitor primer dimers or non-specific product formation. Each LightCycler run contained two negative controls (no DNA added to the reaction), and each DNA sample was quantified in duplicate. A standard curve was established using purified L. infantum DNA; serial dilutions, ranging from 100 to $0.001 \mathrm{ng}$ of DNA parasites, were introduced into reaction tubes in triplicate. The standard curve was generated by LightCycler96 software and used to calculate the equivalent parasite in each sample. The equivalent parasites were calculated considering $200 \mathrm{fg}$ of DNA/Leishmania spp. ${ }^{63-65}$. The data from four animals per group were considered to express the results of qPCR.

Histological evaluations. Spleen and liver were collected, fixed in Bouin's solution, maintained in ethanol $70 \%$, and processed for histological analysis by paraffin inclusion. Briefly, the organs were serially dehydrated in ethanol $(80 \%, 90 \%$, and $100 \%)$ and finally in xylol for at least $12 \mathrm{~h}$. Paraffin substituted the xylol, gradually, at $70{ }^{\circ} \mathrm{C}$, until complete inclusion. The paraffin blocks were cut using a microtome (Leica Biosystems), at $4 \mu \mathrm{m}$ thickness. The sections were collected on two slides previously prepared with poly-L-lysine, stained in Harris' hematoxylin-yellow eosin, and mounted using Permount mounting medium. Spleen and liver from four animals 
were processed and analyzed. Histological analysis from spleen, bone marrow and lymph nodes are the gold standards for identifying Leishmania spp. via observation of intracellular amastigotes. Nevertheless, because of the high sensitivity and the ability to quantify parasite burden, quantitative real-time PCR has been considered the preferred technique for diagnosis. For this reason, parasite quantification was performed by qPCR in this study, and histological analysis evaluated just the general characteristics of the tissues, with qualitative purposes.

Cytokine measurement. Contrary to the hepatic parasitic load, Leishmania infection is not typically self-resolving in the spleen. Thus, cytokine level measurements were carried out only on the spleen samples. For cytokine quantification, a BD Cytometric Bead Array (CBA) Mouse Th1/Th2/Th17 Cytokine Kit was used. First, the BD FACSCalibur flow cytometer was calibrated according to the manufacturer's instructions. To obtain a standard curve, the mouse Th1/Th2/Th17 Cytokines Standard, provided by the kit, was reconstituted and serial dilutions were prepared, followed by the addition of the Mix of Capture Beads and the Mouse Th1/Th2/Th17 PE Detection Reagent, and incubated for $2 \mathrm{~h}$ in the dark at room temperature. Next, the samples were prepared; the previously-weighed spleen was immersed in $0.01 \mathrm{M} \mathrm{PBS}$ at $100 \mu \mathrm{g}$ spleen $/ \mathrm{mL}$. The organ was macerated using ultrasonic equipment (Hielscher UP400St, maximum potency, $10 \mathrm{~s}$ ) and placed in a cold-water bath to avoid heat degradation of the cytokines. The macerated organ was centrifuged and prepared according to the manufacturer's instructions. Briefly, $50 \mu \mathrm{L}$ of the samples were mixed with $50 \mu \mathrm{L}$ of the Mix of Capture Beads and $50 \mu \mathrm{L}$ of the Mouse Th1/Th2/Th17 PE Detection Reagent, then incubated for $2 \mathrm{~h}$ in the dark at room temperature. Subsequently, the samples were washed and resuspended in Wash Buffer provided by the kit, and the samples were analyzed on the BD FACSCalibur cytometer, using FL-3 as the main parameter. For the negative control, a singlet population in the FSC $v s$ SSC dot plot was determined as an R1 region gate. To analyze the samples, the number of events was set to 2,100 within the R1 region gate, ensuring approximately 300 events per Capture Bead, according to the manufacturer's instructions. The data from four animals per group were necessary to quantify the cytokines level using the FCAP Array Software 1.01.

Biochemical analysis. Renal, hepatic and cardiac functions were evaluated from infected and uninfected animals by measuring the creatinine, alanine aminotransferase (ALT), aspartate aminotransferase (AST) and alkaline phosphatase, and creatine kinase MB (CKMB), respectively, using kits for kinetic UV determination. Non-hemolyzed plasma was prepared according to the manufacturer's instructions. The reaction was measured on a Flexstation Microplate Reader at a controlled temperature. From each animal, two reactions were prepared. The reduced volume of blood and plasma obtained from mice allowed all tests to be performed in duplicate from three animals per group.

Statistical analysis. Numerical results from each group were expressed as the average \pm standard error (SE). Statistical analysis was performed with GraphPad Prism software, and the statistically significant difference was determined by the one-way ANOVA test. For cytokine measurements, two-way ANOVA tests were used to examine data with multiples variables, and Tukey's post-test was used to evaluate the intergroup differences. P values less than 0.05 were considered statistically significant.

\section{Data availability}

The datasets analyzed during the current study are available from the corresponding author on reasonable request.

Received: 25 February 2019; Accepted: 16 December 2019;

Published online: 27 December 2019

\section{References}

1. Jain, N., Hwang, T., Franklin, J. M. \& Kesselheim, A. S. Association of the priority review voucher with neglected tropical disease drug and vaccine development. JAMA. 318, 388-389 (2017).

2. Alves, F. et al. Recent development of visceral leishmaniasis treatments: successes, pitfalls, and perspectives. Clin. Microbiol. Rev. 31, e00048-18 (2018)

3. Weng, H. B., Chen, H. X. \& Wang, M. W. Innovation in neglected tropical disease drug discovery and development. Infect. Dis. Poverty. 7, 67 (2018).

4. Croft, S. L. \& Olliaro, P. Leishmaniasis chemotherapy-challenges and opportunities. Clin. Microbiol. Infect. 17, 1478-1483 (2011).

5. Lamotte, S., Späth, G. F., Rachidi, N. \& Prina, E. The enemy within: Targeting host-parasite interaction for antileishmanial drug discovery. PLoS Negl. Trop. Dis. 11, e0005480 (2017).

6. Roatt, B. M. et al. Immunotherapy and immunochemotherapy in visceral leishmaniasis: Promising treatments for this neglected disease. Front. Immunol. 5, 272 (2014).

7. Olivier, M., Gregory, D. J. \& Forget, G. Subversion mechanisms by which Leishmania parasites can escape the host immune response: a signaling point of view. Clin. Microbiol. Rev. 18, 293-305 (2005).

8. Matsuoka, H., Seo, Y., Wakasugp, H., Saito, T. \& Tomoda, H. Lentinan potentiates immunity and prolongs the survival time of some patients. Anticancer Res. 17, 2751-2755 (1997)

9. Honey, K. $\beta$-Glucan conjugate provides protection. Nat. Rev. Drug Discov. 4, 814 (2005).

10. Berner, V. K., duPre, S. A., Redelman, D. \& Hunter, K. W. Microparticulate $\beta$-glucan vaccine conjugates phagocytized by dendritic cells activate both naïve CD4 and CD8 T cells in vitro. Cell. Immunol. 298, 104-114 (2015).

11. Vojtek, B., Mojžǐšová, J., Smrčo, P. \& Drážovská, M. Effects of orally administered $\beta-1,3 / 1,6$-glucan on vaccination responses and immunological parameters in dogs. Food Agric. Immunol. 28, 993-1002 (2017).

12. Kimura, Y., Tojima, H., Fukase, S. \& Takeda, K. Clinical evaluation of sizofilan as assistant immunotherapy in treatment of head and neck cancer. Acta. Otolaryngol. 114, 192-195 (1994).

13. Tanabe, H., Imai, N. \& Takechi, K. Studies on usefulness of postoperative adjuvant chemotherapy with lentinan in patients with gastrointestinal cancer. Nihon Gan Chiryo Gakkai Shi. 25, 1657-1667 (1990).

14. Zhang, Y., Li, S., Wang, X., Zhang, L. \& Cheung, P. C. K. Advances in lentinan: Isolation, structure, chain conformation and bioactivities. Food Hydrocoll. 25, 196-206 (2011). 
15. Vetvicka, V., Vannucci, L., Sima, P. \& Richter, J. Beta glucan: Supplement or drug? From laboratory to clinical trials. Molecules 24, 1251 (2019).

16. Orlean, P. Architecture and biosynthesis of the Saccharomyces cerevisiae cell wall. Genetics 192, 775-818 (2012).

17. Yan, J., Allendorf, D. J. \& Brandley, B. Yeast whole glucan particle (WGP) beta-glucan in conjunction with antitumour monoclonal antibodies to treat cancer. Expert Opin. Biol. Ther. 5, 691-702 (2005).

18. Soto, E. R. \& Ostroff, G. R. Characterization of multilayered nanoparticles encapsulated in yeast cell wall particles for DNA delivery. Bioconjug. Chem. 19, 840-848 (2008).

19. Mirza, Z., Soto, E. R., Dikengil, F., Levitz, S. M. \& Ostroff, G. R. Beta-glucan particles as vaccine adjuvant carriers in Vaccines for invasive fungal infections (eds. Kalkum, M. \& Semis, M.) 143-157 (Springer New York, 2017).

20. Paramera, E. I., Karathanos, V. T. \& Konteles, S. J. Yeast cells and yeast-based materials for microencapsulation in Microencapsulation in the Food Industry. 267-281 (Academic Press, 2014).

21. Taylor, P. R. et al. Dectin-1 is required for $\beta$-glucan recognition and control of fungal infection. Nat. Immunol. 8, 31 (2006).

22. Goodridge, H. S., Wolf, A. J. \& Underhill, D. M. $\beta$-glucan recognition by the innate immune system. Immunol. Rev. 230, 38-50 (2009).

23. Brown, G. D. et al. Dectin-1 Mediates the Biological Effects of $\beta$-Glucans. J. Exp. Med. 197, 1119 LP-1124 (2003)

24. Li, B. et al. Yeast glucan particles activate murine resident macrophages to secrete proinflammatory cytokines via MyD88- and Syk kinase-dependent pathways. Clin. Immunol. 124, 170-181 (2007).

25. Volpato, H. et al. In vitro anti-Leishmania activity of T6 synthetic compound encapsulated in yeast-derived $\beta-(1,3)$-d-glucan particles. Int. J. Biol. Macromol. 119, 1264-1275 (2018).

26. Chan, G. C. F., Chan, W. K. \& Sze, D. M. Y. The effects of beta-glucan on human immune and cancer cells. J. Hematol. Oncol. 2, 25 (2009).

27. Scariot, D. B. et al. Activity and cell-death pathway in leishmania infantum induced by sugiol: Vectorization using yeast cell wall particles obtained from Saccharomyces cerevisiae. Front. Cell. Infect. Microbiol. 9, 208 (2019).

28. Veras, P. S. T. et al. Fusion of Leishmania amazonensis parasitophorous vacuoles with phagosomes containing zymosan particles: Cinemicrographic and ultrastructural observations. Brazilian. J. Med. Biol. Res. 29, 1009-1018 (1996).

29. Veras, P. S. Transfer of zymosan (yeast cell walls) to the parasitophorous vacuoles of macrophages infected with Leishmania amazonensis. J. Exp. Med. 176, 639-646 (2004).

30. Veras, P. S. T. et al. Fusion between large phagocytic vesicles: Targeting of yeast and other particulates to phagolysosomes that shelter the bacterium Coxiella burnetii or the protozoan Leishmania amazonensis in Chinese hamster ovary cells. J. Cell Sci. 107, 3065-3076 (1994).

31. Ponte-Sucre, A. et al. Drug resistance and treatment failure in leishmaniasis: A 21st century challenge. PLoS Negl. Trop. Dis. 11, e0006052 (2017)

32. Boyd, B. J. et al. Successful oral delivery of poorly water-soluble drugs both depends on the intraluminal behavior of drugs and of appropriate advanced drug delivery systems. Eur. J. Pharm. Sci. 137, 104967 (2019).

33. Larrañeta, E., Stewart, S., Ervine, M., Al-Kasasbeh, R. \& Donnelly, R. F. Hydrogels for hydrophobic drug delivery. Classification, synthesis and applications. J. Funct. Biomater. 9,13 (2018).

34. Delalat, B. et al. Targeted drug delivery using genetically engineered diatom biosilica. Nat. Commun. 6, 8791 (2015).

35. Li, J. \& Mooney, D. J. Designing hydrogels for controlled drug delivery. Nat. Rev. Mater. 1, 16071 (2016).

36. Dimov, N., Kastner, E., Hussain, M., Perrie, Y. \& Szita, N. Formation and purification of tailored liposomes for drug delivery using a module-based micro continuous-flow system. Sci. Rep. 7, 12045 (2017).

37. Bosetti, R. Cost-effectiveness of nanomedicine: the path to a future successful and dominant market? Nanomedicine 10, 1851-1853 (2015).

38. Organization, W. H. Visceral leishmaniasis: control strategies and epidemiological situation update in East Africa: report of a WHO bi-regional consultation Addis Ababa, Ethiopia, 9-11 March 2015 (World Health Organization, 2015).

39. de Assis, T. S. M. et al. The direct costs of treating human visceral leishmaniasis in Brazil. Rev. Soc. Bras. Med. Trop. 50, 478-482 (2017).

40. Bhattacharya, P. \& Ali, N. Treatment of visceral leishmaniasis: anomalous pricing and distribution of AmBisome and emergence of an indigenous liposomal amphotericin B, fungisome. J. Parasit. Dis. 40, 1094-1095 (2016).

41. Rousseau, D. et al. Sustained parasite burden in the spleen of Leishmania infantum-infected BALB/c mice is accompanied by expression of MCP-1 transcripts and lack of protection against challenge. Eur. Cytokine Netw. 12, 340-347 (2001).

42. Hermida, Md. E. R. et al. Histological disorganization of spleen compartments and severe visceral leishmaniasis. Front. Cell Infect. Microbiol. 8, 394 (2018).

43. Ren, T. et al. Entrapping of nanoparticles in yeast cell wall microparticles for macrophage-targeted oral delivery of cabazitaxel. Mol. Pharm. 15, 2870-2882 (2018)

44. Bankoti, R. \& Stäger, S. Differential regulation of the immune response in the spleen and liver of mice infected with Leishmania donovani. J. Trop. Med. 2012, 639304 (2012).

45. Bunn, P. T. et al. Tissue requirements for establishing long-term CD4+ t cell-mediated immunity following Leishmania donovani infection. J. Immunol. 192, 3709-3718 (2014).

46. Blackwell, J. M. et al. SLC11A1 (formerly NRAMP1) and disease resistance. Cell. Microbiol. 3, 773-784 (2001).

47. Vidal, S. The Ity/Lsh/Bcg locus: natural resistance to infection with intracellular parasites is abrogated by disruption of the Nramp1 gene. J. Exp. Med. 182, 655-666 (1995).

48. Geller, A., Shrestha, R. \& Yan, J. Yeast-derived $\beta$-glucan in cancer: novel uses of a traditional therapeutic. Int. J. Mol. Sci. 20, 3618 (2019).

49. Novak, M. \& Vetvicka, V. $\beta$-glucans, history, and the present: immunomodulatory aspects and mechanisms of action. J. Immunotoxicol. 5, 47-57 (2008).

50. Moll, H., Flohé, S. \& Röllinghoff, M. Dendritic cells in Leishmania major-immune mice harbor persistent parasites and mediate an antigen-specific T cell immune response. Eur. J. Immunol. 25, 693-699 (1995).

51. Da-Cruz, A. M. et al. T-cell-mediated immune responses in patients with cutaneous or mucosal leishmaniasis: long-term evaluation after therapy. Clin. Diagn. Lab. Immunol. 9, 251-256 (2002).

52. Deng, W., Thiel, B., Tannenbaum, C. S., Hamilton, T. A. \& Stuehr, D. J. Synergistic cooperation between T cell lymphokines for induction of the nitric oxide synthase gene in murine peritoneal macrophages. J. Immunol. 151, 322-329 (1993).

53. Fromm, P. D., Kling, J. C., Remke, A., Bogdan, C. \& Korner, H. Fatal leishmaniasis in the absence of TNF despite a strong Th1 response. Front. Microbiol. 6, 1520 (2015).

54. Bacellar, O. et al. Interleukin-12 restores interferon-gamma production and cytotoxic responses in visceral leishmaniasis. J. Infect. Dis. 173, 1515-1518 (1996).

55. Griewank, K., Gazeau, C., Eichhorn, A. \& von Stebut, E. Miltefosine efficiently eliminates Leishmania major amastigotes from infected murine dendritic cells without altering their immune functions. Antimicrob. Agents Chemother. 54, 652-659 (2010).

56. Parvaneh, N. et al. Visceral leishmaniasis in two patients with IL-12p40 and IL-12R31 deficiencies. Pediatr. Blood Cancer. 64, e26362 (2017).

57. Murray, H. W. Suppression of posttreatment recurrence of experimental visceral leishmaniasis in T-cell-deficient mice by oral miltefosine. Antimicrob. Agents Chemother. 44, 3235-3236 (2000). 
58. Escobar, P., Yardley, V. \& Croft, S. L. Activities of hexadecylphosphocholine (miltefosine), ambisome, and sodium stibogluconate (pentostam) against Leishmania donovani in immunodeficient scid mice. Antimicrob. Agents Chemother. 45, 1872-1875 (2001).

59. Sindermann, H., Engel, K. R., Fischer, C. \& Bommer, W. Oral miltefosine for leishmaniasis in immunocompromised patients: compassionate use in 39 patients with HIV infection. Clin. Infect. Dis. 39, 1520-1523 (2004).

60. Murray, H. W. \& Delph-Etienne, S. Visceral leishmanicidal activity of hexadecylphosphocholine (miltefosine) in mice deficient in $\mathrm{t}$ cells and activated macrophage microbicidal mechanisms. J. Infect. Dis. 181, 795-799 (2000).

61. Cavalcanti, A. S. et al. Parasite load induces progressive spleen architecture breakage and impairs cytokine mRNA expression in Leishmania infantum-naturally infected dogs. PLoS One 10, e0123009 (2015).

62. Kaye, P. M. \& Beattie, L. Lessons from other diseases: granulomatous inflammation in leishmaniasis. Semin. Immunopathol. 38, 249-260 (2016).

63. Mary, C., Faraut, F., Lascombe, L. \& Dumon, H. Quantification of Leishmania infantum DNA by a Real-Time PCR Assay with High Sensitivity. J. Clin. Microbiol. 42, 5249-5255 (2004).

64. Ceccarelli, M., Galluzzi, L., Migliazzo, A. \& Magnani, M. Detection and characterization of Leishmania (Leishmania) and Leishmania (Viannia) by SYBR green-based real-time PCR and high resolution melt analysis targeting kinetoplast minicircle DNA. PLoS One 9, e88845 (2014).

65. Vitale, F. et al. TaqMan-based detection of Leishmania infantum DNA using canine samples. Ann. N. Y. Acad. Sci. 1026, 139-143 (2004).

\section{Acknowledgements}

We would like to thank the COMCAP - UEM, for the support in flow cytometry and histological analysis. This study was supported by Coordination of Improvement of Higher Level Personnel (Capes - Brazil), National Council for Scientific and Technological Development and Sciences without Borders Program (CNPq - Brazil), Araucaria Foundation (Brazil), Financier of Studies and Projects (Finep - Brazil).

\section{Author contributions}

Conception and design: All authors contributed in the concept and design the study. D.S. and H.V. planned the experiments, collected, analyzed, interpreted the data and wrote the manuscript. N.F. and D.L. critically reviewed the manuscript planned, executed, and analyzed the qPCR, the cytokines level measurement, and the histological analysis. M.S., T.U., S.S. and C.N. supported the collect and the biological data analysis. F.R. and A.J. planned, execute the obtaining and provided the T6. A.R. and O.B. proposed, planned and supported the obtention of yeast cell wall particles. C.N. supported, supervised, critically reviewed the manuscript and analyzed the data.

\section{Competing interests}

The authors declare no competing interests.

\section{Additional information}

Supplementary information is available for this paper at https://doi.org/10.1038/s41598-019-56647-w.

Correspondence and requests for materials should be addressed to C.V.N.

Reprints and permissions information is available at www.nature.com/reprints.

Publisher's note Springer Nature remains neutral with regard to jurisdictional claims in published maps and institutional affiliations.

Open Access This article is licensed under a Creative Commons Attribution 4.0 International License, which permits use, sharing, adaptation, distribution and reproduction in any medium or format, as long as you give appropriate credit to the original author(s) and the source, provide a link to the Creative Commons license, and indicate if changes were made. The images or other third party material in this article are included in the article's Creative Commons license, unless indicated otherwise in a credit line to the material. If material is not included in the article's Creative Commons license and your intended use is not permitted by statutory regulation or exceeds the permitted use, you will need to obtain permission directly from the copyright holder. To view a copy of this license, visit http://creativecommons.org/licenses/by/4.0/.

(C) The Author(s) 2019 